\title{
An SIS Epidemic Model with Infective Medium and Feedback Mechanism on Scale-Free Networks
}

\author{
Xiongding Liu, Tao Li*, Yuanmei Wang, Chen Wan, Jing Dong \\ School of Electronics and Information, Yangtze University, Jingzhou, China \\ Email: ^taohust2008@163.com
}

How to cite this paper: Liu, X.D., Li, T., Wang, Y.M., Wan, C. and Dong, J. (2017) An SIS Epidemic Model with Infective Medium and Feedback Mechanism on Scale-Free Networks. Open Access Library Journal, 4: e3598.

https://doi.org/10.4236/oalib.1103598

Received: April 12, 2017

Accepted: May 19, 2017

Published: May 22, 2017

Copyright ( $\odot 2017$ by authors and Open Access Library Inc.

This work is licensed under the Creative Commons Attribution International License (CC BY 4.0).

http://creativecommons.org/licenses/by/4.0/

\begin{abstract}
In this paper, a modified SIS (susceptible-infected-susceptible) model with infective medium and feedback mechanism on scale-free networks is presented. The model is suited to describe some epidemic spreading which are not only transmitting by medium but also spreading between individuals by direct contacts. Considering biological relevance and people's subjective consciousness, we introduce medium and feedback to describe the epidemic spreading. By mathematical analysis, we obtain the epidemic threshold and equilibriums. Simulation shows that the medium parameter can change the threshold, and the bigger it is, the easier epidemic breaks. Feedback parameter cannot change the basic reproductive number, but it can reduce the endemic level and weaken the epidemic spreading.
\end{abstract}

\section{Subject Areas}

Complex Network Models, Simulation/Analytical Evaluation of Communication Systems

\section{Keywords}

Infective Medium, Feedback Mechanism, Basic Reproductive Number, Scale-Free Networks, Equilibrium

\section{Introduction}

As is known to all that epidemic diseases (plague, kala-azar, $\mathrm{H}_{7} \mathrm{~N}_{9}$, SARS, etc.) always bring great harm to the stability of the social and threat individual health. What's more, the rapid development of social and frequent contacts among people contribute to the spread of disease. So, there is account for much attention to study the spreading mechanism and stability for formulating appropriate policies to prevent and control of epidemic diseases [1] [2]. In the early research, 
people considered disease spread model in homogeneous network [3] [4], all individuals in network have the same infected ability in diseases spreading. It's obvious that those models can't completely reflect the realistic feature of the spread of disease. Therefore, the scale-free property is a fundamental discovery in social networks [5] [6]. In the process of researching, many researchers also enrich the epidemic model on scale-free networks. Refs [7] [8] [9] studied the spreading of infections on scale-free networks; these papers found the epidemic threshold on scale-free networks and proved the stability of equilibriums. In order to efficiently control the outbreak of infectious diseases, Chen and Sun [10] firstly succeeded in studying optimal control of an SIRS (susceptible-infected-recovered-susceptible) epidemic model on scale-free networks. In Ref [11] the effect of time delay has been analyzed on scale-free networks in detail.

However, most of researcher works mentioned above, the medium influences on epidemic is not considerate when epidemic spreading. Nevertheless, in the transmission of diseases, such as malaria, dengue fever, those diseases spreading not only by connects between individuals, but contacts between individual and medium. So medium can change the transmission mechanism in epidemic spread. In Ref [12] proposed a SIS (susceptible-infected-susceptible) model with an infected medium on complex network, but the feedback mechanism and the death rate of the medium have not been taken into account. Feedback mechanism considers the initiative response of people when epidemic diseases prevail. Once an epidemic disease outbreaks, people will be more cautious and will reduce contacts with others, the network topology structure will be changed. In this paper, considering the medium and feedback mechanism we focus on a new SIS epidemic model on scale-free networks analyzes in detail.

The rest of the paper is organized as follows: Section 2 derives a SIS model with infective medium and feedback mechanism on scale-free networks. Then, Section 3 obtains two equilibriums and basic reproductive number. Section 4 numerical simulations are performed. Finally, we conclude the paper in Section 5.

\section{Model Formulation}

In order to investigate the efficiency of the medium and feedback mechanism policy, we present an SIS epidemic model with infective medium and feedback mechanism on scale-free networks. In this paper, the epidemic spread model is defined as follows: there are two types of nodes in a network, one is composed of $A$ individuals and the other is composed of $B$ medium. All nodes can only exist in one of the two discrete states, susceptible (i.e. healthy) or infected. We only consider that epidemic diseases can spread between individuals on scale-free networks, individuals and medium spreading by a homogeneous mixing method. In the process of disease transmission, $\lambda$ is the infection rate, an infected individual is cured and become susceptible again with probability $\delta$. The feedback parameter is $\alpha$, it is determined by the fear degree of people to the epidemic disease, $b$ is the natural birth rate, and $\mu$ is the death rate. Here, we assume 
that the birth rate equals the death rate, i.e. $b=\mu$. A healthy person is infected by an infected medium with probability $\gamma_{1}$. A susceptible medium receiving the disease from an infected individual with probability $\gamma_{2}$, the infected medium dies with probability $\beta$. It is also assumed that there is no infection spreading between medium. Let $S_{k}(t)$ and $I_{k}(t)$ be the relative densities of susceptible and infected nodes of degree $k$ at time $t$ respectively. The density of infected medium is $V(t) . \bar{I}(t)=\sum_{i=1}^{n} P(i) I_{i}(t)$ is the density of infected individuals at time $t$.

Based on the above hypotheses, the dynamical mean-field reaction rate equations can be written as

$$
\left\{\begin{array}{l}
\frac{\mathrm{d} S_{k}(t)}{\mathrm{d} t}=b-(1-\alpha \Theta(t)) \lambda k \Theta(t) S_{k}(t)+\delta I_{k}(t)-\mu S_{k}(t)-\gamma_{1} S_{k}(t) V(t) \\
\frac{\mathrm{d} I_{k}(t)}{\mathrm{d} t}=(1-\alpha \Theta(t)) \lambda k \Theta(t)\left(1-I_{k}(t)\right)-\delta I_{k}(t)-\mu I_{k}(t)+\gamma_{1} S_{k}(t) V(t) \\
\frac{\mathrm{d} V(t)}{\mathrm{d} t}=-\beta V(t)+\gamma_{2}(1-V(t)) \bar{I}(t)
\end{array}\right.
$$

where the probability $\Theta(t)$ can be described a link pointing to an infected node, which written as $\Theta(t)=\frac{1}{\langle k\rangle} \sum_{i=1}^{n} \varphi(i) P(i) I_{i}(t)$. where $\langle k\rangle$ is the average degree of the network, i.e. $\langle k\rangle=\sum_{k=1}^{n} k P(k)$, and $\varphi(i)$ is the infectivity of node with degree $i$. Furthermore $S(t)=\sum_{k=1}^{n} P(k) S_{k}, I(t)=\sum_{k=1}^{n} P(k) I_{k}$ are the global average densities of the two states. In view of the practical consideration, we noted that $P(k)>0$ for $k=1,2, \cdots, n$. Those variables obey the normalization condition, i.e. $S_{k}(t)+I_{k}(t)=1$, and the initial conditions for the system (1) satisfy:

$$
S_{k}(0)=1-I_{k}(0)>0, I_{k}(0) \geq 0 \text {, and } \Theta(0)>0 .
$$

\section{Basic Productive Number and Equilibria}

In this section, we reveal some properties of the solutions and the equilibria of system (1).

Theory 1. Consider system (1), Define

$$
R_{0}=\frac{\lambda\left[\left(\beta(\delta+\mu)-\gamma_{1} \gamma_{2}\right)\langle k \varphi(k)\rangle+\gamma_{1} \gamma_{2}\langle\varphi(k)\rangle\langle k\rangle\right]}{(\delta+\mu)\left(\beta(\delta+\mu)-\gamma_{1} \gamma_{2}\right)\langle k\rangle}
$$

The system always exits a disease-free equilibria $E_{0}=(1,0)$ and a unique epidemic equilibrium $E^{*}=\left(S_{k}^{*}, I_{k}^{*}\right)$ if $R_{0}>1$.

Proof. It is easily finding that $E_{0}$ is always an equilibrium of system (1). To obtain the equilibrium $E^{*}$, we need to impose the right side of system (1) to be equal to zero. In other words, the equilibrium $E^{*}=\left(S_{k}^{*}, I_{k}^{*}\right)$ should satisfy

$\left\{\begin{array}{l}b+\delta I_{k}^{*}(t)-\left(1-\alpha \Theta^{*}(t)\right) \lambda k \Theta^{*}(t) S_{k}^{*}(t)-\mu S_{k}^{*}(t)-\gamma_{1} S_{k}^{*}(t) V^{*}(t)=0 \\ -\delta I_{k}^{*}(t)-\mu I_{k}^{*}(t)+\left(1-\alpha \Theta^{*}(t)\right) \lambda k \Theta^{*}(t)\left(1-I_{k}^{*}(t)\right)+\gamma_{1}\left(1-I_{k}^{*}(t)\right) V^{*}(t)=0 \\ -\beta V^{*}(t)+\gamma_{2}\left(1-V^{*}(t)\right) \overline{I^{*}}(t)=0\end{array}\right.$ 
where $\Theta^{*}(t)=\frac{1}{\langle k\rangle} \sum_{k=1}^{n} \varphi(k) P(k) I_{k}^{*}(t)$. We find that

$$
V^{*}(t)=\frac{\gamma_{2} \overline{I^{*}}(t)}{\beta+\gamma_{2} \overline{I^{*}}(t)}, S_{k}^{*}(t)=\frac{(\delta+\mu)\left(\beta+\gamma_{2} \overline{I^{*}}(t)\right)}{\left(1-\alpha \Theta^{*}\right) \lambda k \Theta^{*}+\gamma_{1} \gamma_{2} \overline{I^{*}}(t)} I_{k}^{*}(t)
$$

Inserting Equation into Equation, we have

$$
I_{k}^{*}(t)=\frac{\left(\beta+\gamma_{2} \overline{I^{*}}(t)\right) \lambda k\left[1-\alpha \Theta^{*}(t)\right] \Theta^{*}(t)+\gamma_{1} \gamma_{2} \overline{I^{*}}(t)}{(\delta+\mu)\left(\beta+\gamma_{2} \overline{I^{*}}(t)\right)+\left(\beta+\gamma_{2} \overline{I^{*}}(t)\right) \lambda k\left[1-\alpha \Theta^{*}(t)\right] \Theta^{*}(t)+\gamma_{1} \gamma_{2} \overline{I^{*}}(t)}
$$

Let

$$
\begin{gathered}
F\left(\overline{I^{*}}, \Theta^{*}\right)=\left(\beta+\gamma_{2} \overline{I^{*}}(t)\right) \lambda k\left[1-\alpha \Theta^{*}(t)\right] \Theta^{*}(t)+\gamma_{1} \gamma_{2} \overline{I^{*}}(t) \\
G\left(\overline{I^{*}}, \Theta^{*}\right)=(\delta+\mu)\left(\beta+\gamma_{2} \overline{I^{*}}(t)\right)+\left(\beta+\gamma_{2} \overline{I^{*}}(t)\right) \lambda k\left[1-\alpha \Theta^{*}(t)\right] \Theta^{*}(t)+\gamma_{1} \gamma_{2} \overline{I^{*}}(t) \\
\text { Then } I_{k}(t)=\frac{F\left(\overline{I^{*}}, \Theta^{*}\right)}{G\left(\overline{I^{*}}, \Theta^{*}\right)} .
\end{gathered}
$$

Therefore, a self-consistency equation can be obtained

$$
\Theta^{*}(t)=\frac{1}{\langle k\rangle} \sum_{i=1}^{n} \varphi(k) P(k) \frac{F\left(\overline{I^{*}}, \Theta^{*}\right)}{G\left(\overline{I^{*}}, \Theta^{*}\right)}=W\left(\Theta^{*}\right)
$$

Obviously, when $I_{k}=0$, so $\bar{I}=0, \Theta(t)(t)=0$, it is a zero solution of (3). If there exist another positive solution $\Theta(t)>0$, it follows that

From the following form of $\overline{I^{*}}$

$$
\left.\frac{\mathrm{d} W\left(\Theta^{*}\right)}{\mathrm{d} \Theta^{*}}\right|_{\Theta^{*}=0}>1
$$

$$
\begin{aligned}
& \overline{I^{*}}(t)=\sum_{k=1}^{n} P(k) I_{k}^{*}(t) \\
& =\sum_{k=1}^{n} P(k) \frac{\left(\beta+\gamma_{2} \overline{I^{*}}(t)\right) \lambda k\left[1-\alpha \Theta^{*}(t)\right] \Theta^{*}(t)+\gamma_{1} \gamma_{2} \overline{I^{*}}(t)}{(\delta+\mu)\left(\beta+\gamma_{2} \overline{I^{*}}(t)\right)+\left(\beta+\gamma_{2} \overline{I^{*}}(t)\right) \lambda k\left[1-\alpha \Theta^{*}(t)\right] \Theta^{*}(t)+\gamma_{1} \gamma_{2} \overline{I^{*}}(t)}
\end{aligned}
$$

We get that $\left.\frac{\mathrm{d} I^{*}}{\mathrm{~d} \Theta^{*}}\right|_{\Theta^{*}=0}=\frac{\beta \lambda\langle k\rangle}{\beta(\delta+\mu)-\gamma_{1} \gamma_{2}}$.

Let

$$
\begin{gathered}
F=\left(\beta+\gamma_{2} \overline{I^{*}}(t)\right) \lambda k\left[1-\alpha \Theta^{*}(t)\right] \Theta^{*}(t)+\gamma_{1} \gamma_{2} \overline{I^{*}}(t) \\
G=(\delta+\mu)\left(\beta+\gamma_{2} \overline{I^{*}}(t)\right)+\left(\beta+\gamma_{2} \overline{I^{*}}(t)\right) \lambda k\left[1-\alpha \Theta^{*}(t)\right] \Theta^{*}(t)+\gamma_{1} \gamma_{2} \overline{I^{*}}(t)
\end{gathered}
$$

Then, we have computed that

$$
\begin{aligned}
\left.\frac{\mathrm{d} W(\Theta)}{\mathrm{d} \Theta}\right|_{\Theta=0} & =\left.\frac{1}{\langle k\rangle} \sum_{i=1}^{n} \varphi(i) P(i) \frac{\left(\frac{\partial F}{\partial \bar{I}} \cdot \frac{\partial \bar{I}}{\partial \Theta}+\frac{\partial F}{\partial \Theta}\right) G-\left(\frac{\partial G}{\partial \bar{I}} \cdot \frac{\partial \bar{I}}{\partial \Theta}+\frac{\partial G}{\partial \Theta}\right) F}{G^{2}}\right|_{\Theta=0} \\
& =\frac{\lambda\left[\left(\beta(\delta+\mu)-\gamma_{1} \gamma_{2}\right)\langle k \varphi(k)\rangle+\gamma_{1} \gamma_{2}\langle\varphi(k)\rangle\langle k\rangle\right]}{(\delta+\mu)\left(\beta(\delta+\mu)-\gamma_{1} \gamma_{2}\right)\langle k\rangle}>1
\end{aligned}
$$


We can get the basic reproduction number

$$
R_{0}=\frac{\lambda\left[\left(\beta(\delta+\mu)-\gamma_{1} \gamma_{2}\right)\langle k \varphi(k)\rangle+\gamma_{1} \gamma_{2}\langle\varphi(k)\rangle\langle k\rangle\right]}{(\delta+\mu)\left(\beta(\delta+\mu)-\gamma_{1} \gamma_{2}\right)\langle k\rangle} .
$$

The unique nontrivial solution exist when $R_{0}>1$. Therefore, the epidemic threshold can be defined by:

$$
\lambda_{c}=\frac{(\delta+\mu)\left(\beta(\delta+\mu)-\gamma_{1} \gamma_{2}\right)\langle k\rangle}{\left(\beta(\delta+\mu)-\gamma_{1} \gamma_{2}\right)\langle k \varphi(k)\rangle+\gamma_{1} \gamma_{2}\langle\varphi(k)\rangle\langle k\rangle}
$$

Inserting the nontrivial solution of (4) into (3), we can get $I_{k}^{*}$. Then we can obtain $0<S_{k}^{*}<1,0<I_{k}^{*}<1$ for $k=1,2, \cdots, n$. So, there is a unique epidemic equilibrium $E^{*}=\left(S_{k}^{*}, I_{k}^{*}\right)$ if $R_{0}>1$. The proof is completed.

Remark

(1) The epidemic threshold $\lambda_{c}$ is obtained by Equation (5). The epidemic threshold depends on the fluctuations of the degree distribution and some model parameters. Interestingly, the feedback mechanism parameter $\alpha$ cannot change the epidemic threshold $\lambda_{c}$.

(2) If $\delta=1, \mu=0$ and, $\alpha=0$ then the system (1) becomes the SIS model with the influence of the infective vector. Then the epidemic threshold

$$
\lambda_{c}=\frac{\left(1-\gamma_{1} \gamma_{2}\right)\langle k\rangle}{\left(1-\gamma_{1} \gamma_{2}\right)\langle k \varphi(k)\rangle+\gamma_{1} \gamma_{2}\langle\varphi(k)\rangle\langle k\rangle}
$$

which consists with Ref [13]. The infected medium is affected the epidemic threshold.

(3) If $\varphi(k)=k, \delta=1, \mu=0$ and $\alpha=0$ then the system (1) becomes the SIS model with the influence of the infective medium. Then the epidemic threshold

$$
\lambda_{c}=\frac{\left(1-\gamma_{1} \gamma_{2}\right)\langle k\rangle}{\gamma_{1} \gamma_{2}\left(\langle k\rangle^{2}-\left\langle k^{2}\right\rangle\right)+\left\langle k^{2}\right\rangle}
$$

which consists with Ref [14]. The infected medium is affected the epidemic threshold.

(4) If $\varphi(k)=k, \delta=1, \mu=0, \alpha=0$ and $\gamma_{1} \gamma_{2}=0$, then the system (1) becomes the standard SIS model without the influence of the infective medium. Then the epidemic threshold $\lambda_{c}=\langle k\rangle /\left\langle k^{2}\right\rangle$, which consists with Ref [15]. The epidemic threshold only correlated with network topology.

(5) If $\varphi(k)=k$ and $\gamma_{1} \gamma_{2}=0$ then the system (1) becomes the standard SIS model with feedback mechanism, which consists with Ref [16].

From above we discussed, the simulation can show our result in detail. The disease-free equilibrium $E_{0}$ is stable when $R_{0}<1$; and the disease is permanent on the network when $R_{0}>1$. The medium can influence the epidemic outbreak, feedback parameter can't change the threshold but can weaken the epidemic outbreak.

\section{Numerical Simulations}

For epidemic spreading with infected medium and feedback mechanism on scale-free network, we know that the stability of the disease-free equilibrium and 
locally equilibrium depend on threshold. In this section, we study by RungeKutta numerical simulations to discuss the effects of some parameters on the epidemic threshold. We consider the system (1) on a scale-free network with the degree distribution $P(k)=\xi k^{-r}$ where $r=3$ and $\sum_{i=1}^{n} P(i)=1, n=1000$. We set the initial density of infected nodes to be 0.9 , like studies [17]. Birth rate is proportional to death rate, which keeps the population stable from beginning.

In Figure 1. Where we choose $\delta=0.2, \mu=0.4, \beta=0.4, \gamma_{2}=0.3, \varphi(k)=k$. It illustrates that medium has great influence on epidemic spreading. We can see that the easier epidemic outbreak as the spreading rate of infected medium increase. The infected mediums contribute to epidemic outbreak, it satisfies our common sense.

In Figure 2, the parameters are chosen as

$l=0.4, \lambda=0.1, \alpha=0.1, \delta=0.6, \gamma_{1}=0.01, \gamma_{2}=0.4, \quad \beta=0.4, \quad R_{0}=0.459$, with the infected initial value $I_{100}(0)=0.1$. We can see that when $R_{0}<1, I_{k}$ approach to zero, i.e., the infectious individuals will ultimately disappear.

In Figure 3, the parameters are chosen as

$l=0.3, \lambda=0.35, \alpha=0.1, \delta=0.25, \gamma_{1}=0.2, \quad \beta=0.4, \quad \gamma_{2}=0.2, \quad R_{0}=3.45$. We can see that when $R_{0}>1, I_{k}$ grows to a stable value, i.e., the epidemic disease is permanent and the number of infected individual will converge to a positive value.

In Figure 4, the parameters are chosen as

$l=0.4, \lambda=0.1, \delta=0.6, \gamma_{1}=0.01, \gamma_{2}=0.4, \quad \beta=0.4, \quad R_{0}=0.459, \quad \alpha=5,3,1,0.1$ from bottom to top. We can see that the corresponding $I_{100}$ decreases significantly as the feedback parameter increases $\alpha$, i.e., a larger feedback parameter can weaken the spreading of disease.

In Figure 5, the parameters are chosen as $l=0.4, \lambda=0.4, \delta=0.2, \gamma_{1}=0.2, \gamma_{2}=0.2, \beta=0.4, \quad \alpha=5,3,1,0.1$, from bottom to top, $R_{0}=1.398$. We can see that the $I_{100}$ corresponding decreases significantly as the feedback parameter $\alpha$ increases, i.e., a larger feedback parameter can reduce the endemic level.

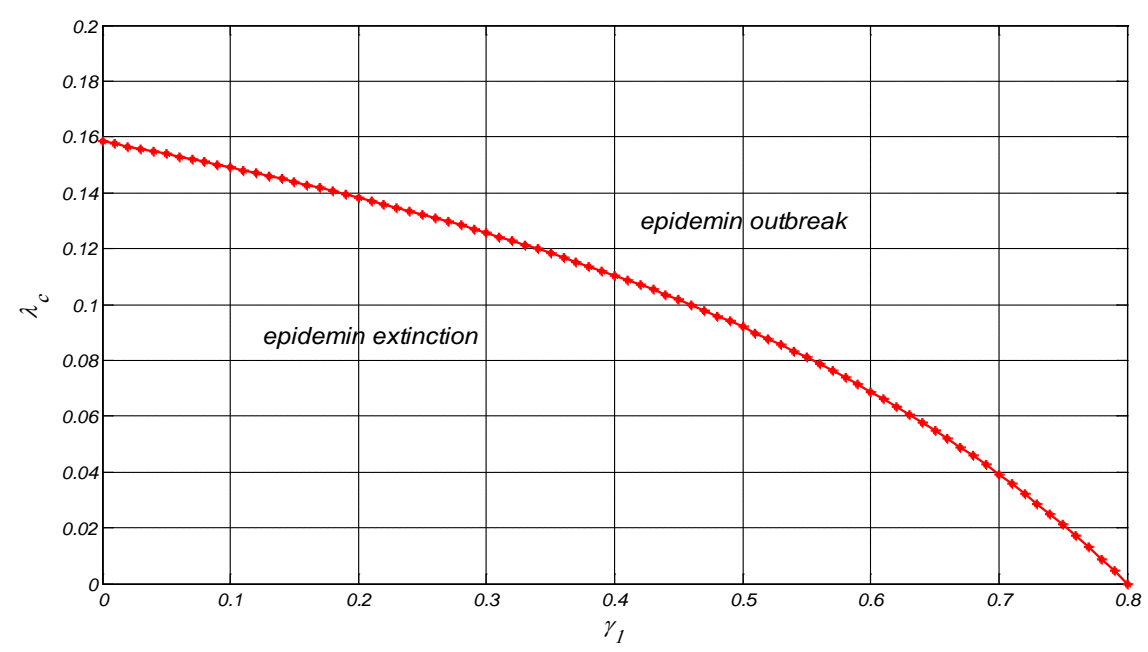

Figure 1. The influence of medium influences the epidemic outbreak. 


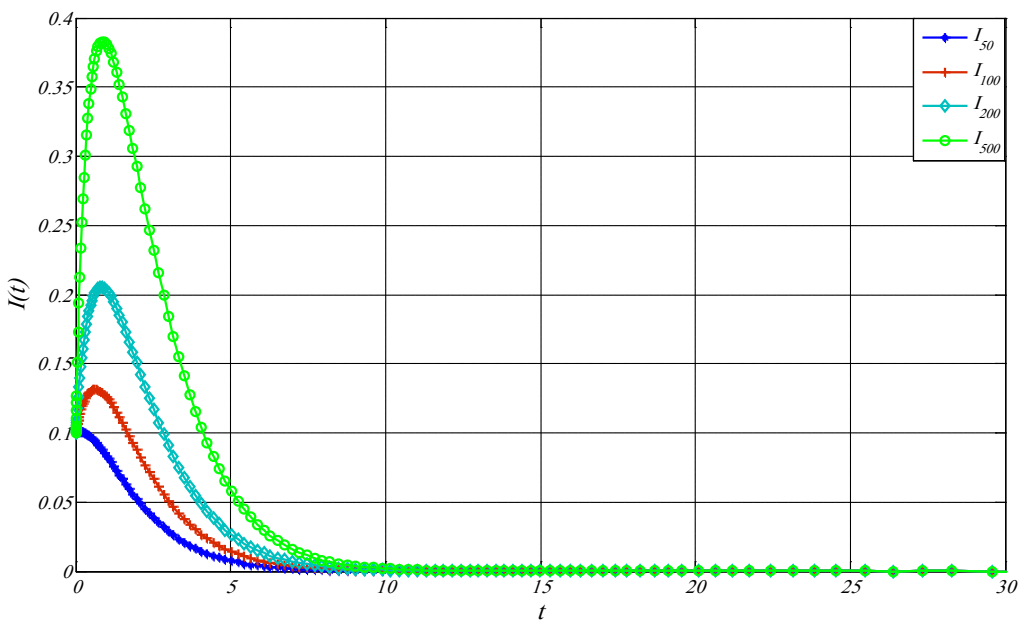

Figure 2. The time series of system (1) with $R_{0}<1$ and initial values $I_{(k)}(0)=0.1, k=50,100,200,500$.

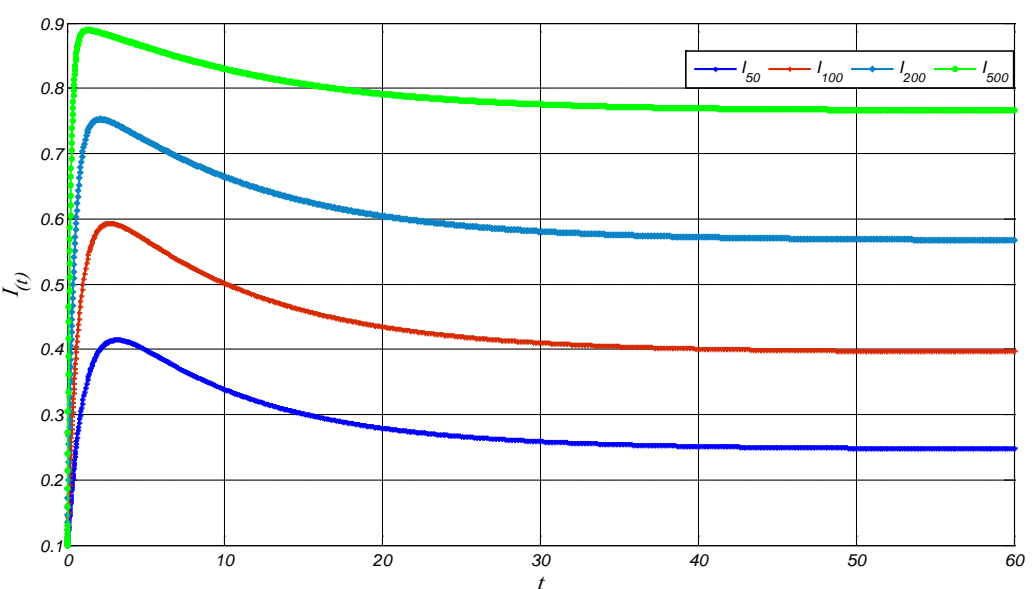

Figure 3. The time series of system (1) with $R_{0}>1$ and initial values $I_{(k)}(0)=0.1, k=50,100,200,500$.

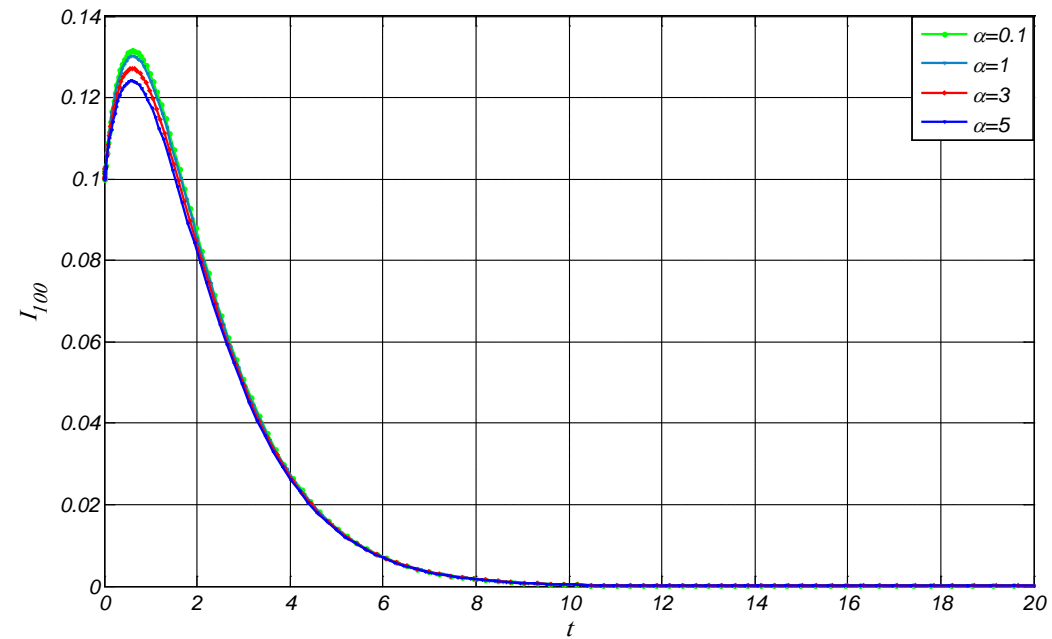

Figure 4. The prevalence $I_{100}$ versus $t$ with $R_{0}=0.459$ to different $\alpha$ with identical initial value $I_{100}(0)=0.1$. 


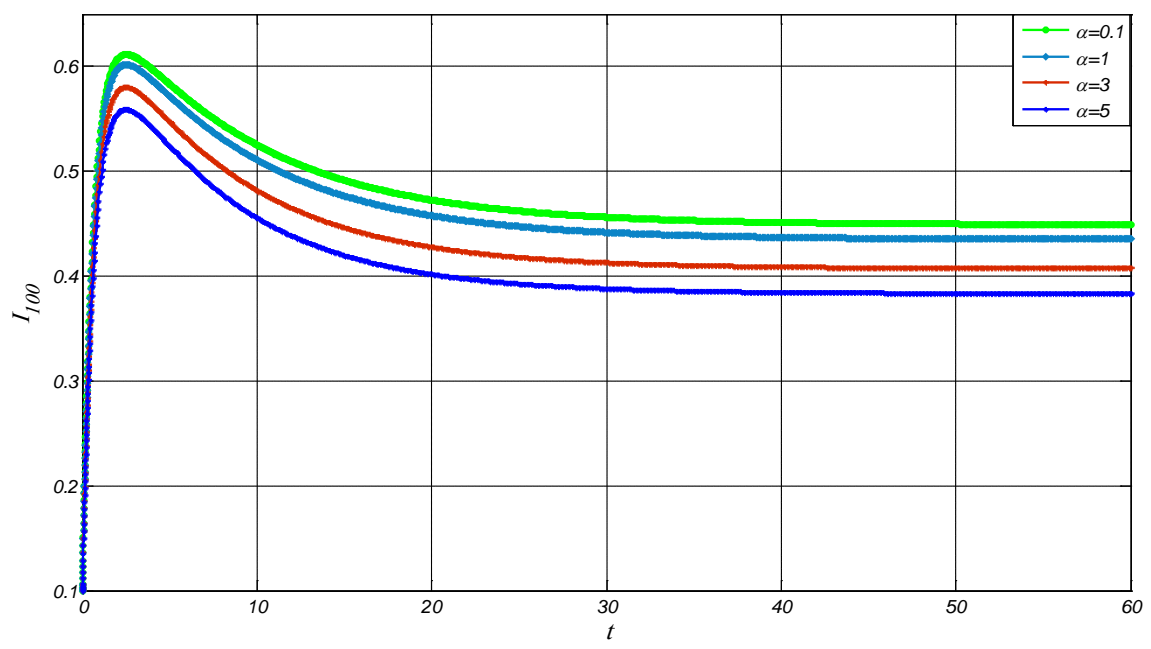

Figure 5. The prevalence $I_{100}$ versus $t$ with $R_{0}=1.389$ to different $\alpha$ with identical initial value $I_{100}(0)=0.1$.

\section{Conclusion}

To study the influence of the spread of the disease and the topology of the network, we proposed a modified SIS model on scale-free network with infected medium and feedback mechanism. By using some mathematical analytic method, we found the basic reproduction number $R_{0}$ and equilibriums. Theoretical results indicate that the basic reproduction number is dependent on the topology of the underlying networks. The simulation has shown that the disease-free equilibrium $E_{0}$ is stable when $R_{0}<1$; and the disease is permanent on the network when $R_{0}>1$. Here the rigorous proof that we could not complete will be discussed in the future. In addition, we added the medium into the normal SIS model, it shows medium contributes to epidemic spreading. As for feedback mechanism, we take note of that epidemic threshold bears no relation to the feedback parameter, but formulate shows that it can accelerate the extinction of the disease and weaken the endemic level, which complies with our realistic sense. The study may give us valuable guiding in effectively predicting and preventing epidemic spreading.

\section{Acknowledgements}

This work is supported by the National Natural Science Foundation of China under Grant 61672112 and Project in Hubei province department of education under Grant B2016036.

\section{References}

[1] Dye, C. and Gay, N. (2003) Modeling the SARS Epidemic. Science, 300, 1884-1885. https://doi.org/10.1126/science.1086925

[2] Epstein, J. (2009) Modeling to Contain Pandemics. Nature, 460, 687.

[3] Pastor-Satorras, R. and Vespignani, A. (2004) Evolution and Structures of the Internet: A Statistical Physics Approach. Cambridge University Press, Cambridge. 
https://doi.org/10.1017/CBO9780511610905

[4] Watts, D.J. and Strogats, S.H. (1998) Collective Dynamics of 'Small-World' Networks. Nature (London), 393, 440-442.

[5] Barabási, A.-L. and Albert, R. (1999) Emergence of Scaling in Random Networks. Science, 286, 509.

[6] Boccaletti, S., Latora, V., Moreno, Y., et al. (2006) Complex Networks: Structure and Dynamics. Physics Reports, 424,175. https://doi.org/10.1016/j.physrep.2005.10.009

[7] Li, C.H. and Tsai, C.C. (2014) Analysis of Epidemic Spreading of an SIRS Model in Complex Heterogeneous Networks. Communications in Nonlinear Science and Numerical Simulation, 19, 1042-1054. https://doi.org/10.1016/j.cnsns.2013.08.033

[8] Moreno, Y. and Pastor-Satorras, R. (2002) Epidemic Outbreaks in Complex Heterogeneous Networks. European Physical Journal B-Condensed Matter and Complex Systems, 26, 521-529.

[9] Li, T. and Wang, Y. (2014) Spreading Dynamics of a SIQRS Epidemic Model on Scale-Free Networks. Communications in Nonlinear Science and Numerical Simulation, 19, 686-692. https://doi.org/10.1016/j.cnsns.2013.07.010

[10] Chen, L. and Sun, J. (2014) Global Stability and Optimal Control of an SIRS Epidemic Model on Heterogeneous Networks. Physica A, 410, 196-204. https://doi.org/10.1016/j.physa.2014.05.034

[11] Liu, Q.M. and Deng, C.S. (2014) The Analysis of an Epidemic Model with Time Delay on Scale-Free Networks. Physica A: Statistical Mechanics and Its Applications, 410, 79-87. https://doi.org/10.1016/j.physa.2014.05.010

[12] Yang, M., Chen, G. and Fu, X. (2011) A Modified SIS Model with an Infective Medium on Complex Networks and Its Global Stability. Physica A: Statistical Mechanics and Its Applications, 390, 2408-2413. https://doi.org/10.1016/j.physa.2011.02.007

[13] Kang, H. and Fu, X. (2015) Epidemic Spreading and Global Stability of an SIS Model with an Infective Vector on Complex Networks. Communications in Nonlinear Science and Numerical Simulation, 27, 30-39. https://doi.org/10.1016/j.cnsns.2015.02.018

[14] Yang, M., Chen, G. and Fu, X. (2011) A Modified SIS Model with an Infective Medium on Complex Networks and Its Global Stability. Physica A: Statistical Mechanics and Its Applications, 390, 2408-2413. https://doi.org/10.1016/j.physa.2011.02.007

[15] Pastor-Satorras, R. and Vespignani, A. (2001) Epidemic Dynamics and Endemic States in Complex Networks. Physical Review E, 63, Article ID: 066117. https://doi.org/10.1103/PhysRevE.63.066117

[16] Sun, J.Z.J. (2014) Stability Analysis of an SIS Epidemic Model with Feedback Mechanism on Networks. Physica A, 394, 24-32.

[17] Li, T., Liu, X.D. and Wu, J. (2016) An Epidemic Spreading Model on Adaptive Scale-Free Networks with Feedback Mechanism. Physica A, 450, 649-656. https://doi.org/10.1016/j.physa.2016.01.045 
Submit or recommend next manuscript to OALib Journal and we will provide best service for you:

- Publication frequency: Monthly

- 9 subject areas of science, technology and medicine

- Fair and rigorous peer-review system

- Fast publication process

- Article promotion in various social networking sites (LinkedIn, Facebook, Twitter, etc.)

- Maximum dissemination of your research work

Submit Your Paper Online: Click Here to Submit

Or Contact service@oalib.com 